\title{
Extended Oligopolies with Pollution Penalties and Rewards
}

\author{
Akio Matsumoto $\left(\mathrm{D},{ }^{1}\right.$ Ferenc Szidarovszky, ${ }^{2}$ and Hirokazu Takizawa ${ }^{1}$ \\ ${ }^{1}$ Department of Economics, International Center for Further Development of Dynamic Economic Research, \\ Chuo University, 742-1, Higashi-Nakano, Hachioji, Tokyo 192-0393, Japan \\ ${ }^{2}$ Department of Applied Mathematics, University of Pécs, Ifjúság u. 6., 7624 Pécs, Hungary \\ Correspondence should be addressed to Akio Matsumoto; akiom@tamacc.chuo-u.ac.jp
}

Received 29 December 2017; Revised 1 May 2018; Accepted 14 May 2018; Published 21 June 2018

Academic Editor: Douglas R. Anderson

Copyright (C) 2018 Akio Matsumoto et al. This is an open access article distributed under the Creative Commons Attribution License, which permits unrestricted use, distribution, and reproduction in any medium, provided the original work is properly cited.

\begin{abstract}
An extended $n$-firm oligopoly with product differentiation is considered. It is assumed that the government selects an emission standard for the industry and based on the output and technology of each firm it selects a maximum allowed amount of emission for each firm. If the actual amount is higher than the allowed maximum, then the firm has to pay a constant multiple of the excess to the government; otherwise it is rewarded similarly based on the saved emission amount. The existence of the unique interior equilibrium is first proved, and then the effect of the level of penalty or reward and that of the emission standard on the industry output and therefore on the total emission level is also examined. Time delay is introduced into the penalties the firms have to pay and into the rewards the firms receive. In analyzing the stability of the equilibrium both discrete and continuous time scales are considered. For mathematical simplicity the case of symmetric firms is analyzed. In the discrete case the various values of the delay length are examined. The equilibrium is stable if either the total industry output is sufficiently large or the common speed of adjustment of the firms is sufficiently small. In the continuous case, either the equilibrium is always stable or stability occurs if the delay is sufficiently small and at the critical value Hopf bifurcation occurs.
\end{abstract}

\section{Introduction}

In classical duopoly models the firms are competing in a market and their production outputs or prices are the decision variables. Their profits are the differences of their selling revenues and production costs. These classical models have been extended in many different ways including the consideration of environmental issues. In this direction, the effect of different environmental regulation policies has been investigated by many researchers ([1-3] among others). Mostly single firms were considered in relation to environmental $\mathrm{R} \& \mathrm{D}$, and very few works were devoted to the extension of oligopoly models in this direction. Montero [3] examined the effect of R\&D investment for pollution abatement technology with different environmental policies in duopolistic product markets. Static models were investigated in earlier stages; the existence of equilibrium in Cournot oligopoly with pollution treatment cost sharing was proved by Okuguchi and Szidarovszky [4], whose work was further extended by including emission standard and R\&D in the oligopoly model [5]. If the government is unable to assess the individual emission levels of the different firms, then it can measure only the total level of pollution, and ambient charges are introduced. In this policy [6] the government defines a cut-off for the total pollution level and, regardless of the specific emission level of each firm, all are equally punished or rewarded. Ganguli and Raju [7] demonstrated that in a Bertrand duopoly increasing ambient charges could lead to greater pollution; however Raju and Ganguli [8] showed the opposite effect in a Cournot duopoly framework when the increase of ambient charges reduces pollution. This result was further generalized by Matsumoto et al. [9] for $n$-firm oligopolies where the stability of the dynamic model with naive expectation was also examined. If the government is familiar with the used technology and production output of each firm, then it is able to assess the proportion of each firm from the total pollution level. Therefore each firm can be punished or rewarded according to its assessed individual emission level compared to its allowed proportional maximum from the government defined cut-off threshold. In this paper this idea will be 
elaborated. After the formulation of the mathematical model the existence of the static equilibrium will be proved. Then the effect of penalty and reward parameters on the industry output and the total pollution level will be investigated. Assuming gradient adjustment of the firms and introducing time delay into the penalties and the rewards, dynamic models will be developed with both discrete and continuous time scales, and the stability conditions of the equilibrium will be derived, analyzed, and compared.

\section{Model and Cournot-Nash Equilibrium}

Consider $n$ firms in an oligopoly with differentiated products. Let $q_{k}$ be output of firm $k$. The price of the product of firm $k$ is seen as

$$
p_{k}=a_{k}-q_{k}-\gamma_{k} \sum_{i \neq k}^{n} q_{i}
$$

with $n \geq 2$ and $a_{k}>0$ and $0<\gamma_{k}<1$. Firm $k$ emits pollution $e_{k} q_{k}$ in connection with its production with $e_{k}>0$. The value of $e_{k}$ is technology-dependent and assumed to be fixed. (In our next project we will consider the profitability of technology changes with their additional costs and environmental benefits.) The government can measure the total emission quantity and has an exogenously selected environmental standard $\bar{E}$. So the maximum allowed emission of firm $k$ is clearly

$$
\frac{e_{k} q_{k}}{\sum_{i=1}^{n} e_{i} q_{i}} \bar{E}
$$

If a firm exceeds this amount then it has to pay a penalty of $m$ times the exceeded amount, and if its emission amount is below the maximum allowed amount, then the firm is rewarded by $m$ times the saved emission amount. With (2), the payoff of firm $k$ becomes

$$
\pi_{k}=\left(p_{k}-c_{k}\right) q_{k}-m\left(e_{k} q_{k}-\frac{e_{k} q_{k}}{\sum_{i=1}^{n} e_{i} q_{i}} \bar{E}\right)
$$

where $c_{k}$ is the marginal cost of firm $k$. Substituting (1) into (3) yields

$$
\begin{aligned}
\pi_{k}= & \left(a_{k}-q_{k}-\gamma_{k} \sum_{i \neq k}^{n} q_{i}-c_{k}\right) q_{k} \\
& -\left(m e_{k}-\frac{m e_{k}}{\sum_{i=1}^{n} e_{i} q_{i}} \bar{E}\right) q_{k} .
\end{aligned}
$$

The first term corresponds to revenue and production cost and the second term refers to emission penalty or reward. Assuming interior optimum, the first order condition implies that

$$
\begin{aligned}
\frac{\partial \pi_{k}}{\partial q_{k}}= & \left(a_{k}-2 q_{k}-\gamma_{k} \sum_{i \neq k}^{n} q_{i}-c_{k}\right) \\
& -\left(m e_{k}-\frac{m e_{k} \sum_{i \neq k}^{n} e_{i} q_{i}}{\left(\sum_{i=1}^{n} e_{i} q_{i}\right)^{2}} \bar{E}\right)=0 .
\end{aligned}
$$

Notice that (5) strictly decreases in $q_{k}$ with fixed values of $q_{i}(i \neq k)$. At $q_{k}=0$, its value is

$$
a_{k}-\gamma_{k} \sum_{i \neq k}^{n} q_{i}-c_{k}-m e_{k}+\frac{m e_{k}}{\sum_{i \neq k}^{n} e_{i} q_{i}} \bar{E} .
$$

If this value is nonpositive, then $q_{k}=0$ is optimum, which is not interior. As $q_{k} \rightarrow \infty$, the value of (5) tends to $-\infty$, so there is always a unique best response.

For mathematical simplicity, let us assume symmetric firms in the sense that firms have identical substitutability and technology in emission production.

Assumption 1. $\gamma_{1}=\gamma_{2}=\cdots=\gamma_{n}=\gamma$ and $e_{1}=e_{2}=\cdots=e_{n}=$ e.

Since the maximum prices $\alpha_{k}$ are different, the firms might have different output levels. Then (5) becomes

$$
\begin{aligned}
& a_{k}-2 q_{k}-\gamma \sum_{i=1}^{n} q_{i}+\gamma q_{k}-c_{k}-m e+\frac{m\left(\sum_{i=1}^{n} q_{i}-q_{k}\right)}{\left(\sum_{i=1}^{n} q_{i}\right)^{2}} \bar{E} \\
& \quad=0
\end{aligned}
$$

and, with notation $Q=\sum_{i=1}^{n} q_{i}$, we have

$$
a_{k}-\gamma Q-c_{k}-m e+\frac{m \bar{E}}{Q}=q_{k}\left(2-\gamma+\frac{m \bar{E}}{Q^{2}}\right) \text {. }
$$

So

$$
q_{k}=\frac{\left(a_{k}-c_{k}-m e\right) Q^{2}-\gamma Q^{3}+m \bar{E} Q}{(2-\gamma) Q^{2}+m \bar{E}} .
$$

By adding these equations for $k=1,2, \ldots, n$ and dividing by Q,

$$
\begin{aligned}
(2-\gamma) Q^{2}+m \bar{E}= & \left(\sum_{k=1}^{n} a_{k}-\sum_{k=1}^{n} c_{k}-n m e\right) Q-n \gamma Q^{2} \\
& +n m \bar{E}
\end{aligned}
$$

which is a quadratic equation for $Q$,

$$
\begin{gathered}
{[(2-\gamma)+n \gamma] Q^{2}-\left(\sum_{k=1}^{n} a_{k}-\sum_{k=1}^{n} c_{k}-n m e\right) Q} \\
-(n-1) m \bar{E}=0 .
\end{gathered}
$$

At $Q=0$, the left-hand side is negative and as $Q \rightarrow \infty$, it converges to $+\infty$, so there is real root. Since the constant term is negative, one root is positive and the other is negative. So only the positive root has economic meaning. Then the corresponding equilibrium levels of the firms are given by (9).

\section{Effect of Penalty or Reward on Pollution Levels}

The penalty factor $m$ and the emission standard $\bar{E}$ are the strategic (or fiscal-policy) variables of the government and 
should be determined so as to maximize a social welfare function which includes the sum of the firms' profits, consumer's surplus, and any technological external effects. (Determining the optimal values of these variables could be a research subject of a next paper.) In this study, however, we treat them as exogenously determined parameters and analyze how the total production level (and therefore the total emission level) depends on $m$ and $\bar{E}$.

Considering $Q$ as a function of $m$, and implicitly differentiating (11) with respect to $m$, we have

$$
\begin{aligned}
& {[(2-\gamma)+n \gamma] 2 Q \frac{d Q}{d m}+n e Q} \\
& \quad-\left(\sum_{k=1}^{n} a_{k}-\sum_{k=1}^{n} c_{k}-n m e\right) \frac{d Q}{d m}+(1-n) \bar{E}=0
\end{aligned}
$$

implying that

$$
\begin{aligned}
& \frac{d Q}{d m} \\
& \quad=\frac{-n e Q+(n-1) \bar{E}}{2 Q[(2-\gamma)+n \gamma]-\left(\sum_{k=1}^{n} a_{k}-\sum_{k=1}^{n} c_{k}-n m e\right)} .
\end{aligned}
$$

Based on (11), the denominator can be rewritten as

$$
Q[(2-\gamma)+n \gamma]+\frac{(n-1) m \bar{E}}{Q}
$$

which is positive. So the sign of $d Q / d m$ depends on the sign of the numerator. The first term is negative; the second term is positive. Using again (11), we see that the $m$-multiple of the numerator equals

$$
-\left(\sum_{k=1}^{n} a_{k}-\sum_{k=1}^{n} c_{k}\right) Q+[(2-\gamma)+n \gamma] Q^{2} .
$$

It is reasonable to assume that the first term is negative. Therefore $d Q / d m>0$ if and only if

$$
Q>\frac{\sum_{k=1}^{n} a_{k}-\sum_{k=1}^{n} c_{k}}{(2-\gamma)+n \gamma} .
$$

Let $\bar{Q}$ denote the right-hand side of this inequality and $P(Q)$ again the left-hand side of (11); then this is the case when $P(\bar{Q})<0$ meaning that

$$
\begin{aligned}
& \bar{E} \\
& >\frac{[(2-\gamma)+n \gamma] \bar{Q}^{2}-\left(\sum_{k=1}^{n} a_{k}-\sum_{k=1}^{n} c_{k}-n m e\right) \bar{Q}}{(n-1) m} .
\end{aligned}
$$

It is easy to see that the numerator is positive. This inequality means that increase in the value of $m$ has an increasing effect on the total industry output as well as in the total emission level if the emission standard $\bar{E}$ is sufficiently large. Otherwise the opposite effect can be observed.
Next we examine the effect of increasing the value of $\bar{E}$. Considering now $Q$ as function of $\bar{E}$ and implicitly differentiating (11) we have

$$
\begin{aligned}
& {[(2-\gamma)+n \gamma] 2 Q \frac{d Q}{d \bar{E}}-\left(\sum_{k=1}^{n} a_{k}-\sum_{k=1}^{n} c_{k}-n m e\right) \frac{d Q}{d \bar{E}}} \\
& +(1-n) m=0
\end{aligned}
$$

showing that

$$
\begin{aligned}
& \frac{d Q}{d \bar{E}} \\
& \quad=\frac{(n-1) m}{2 Q[(2-\gamma)+n \gamma]-\left(\sum_{k=1}^{n} a_{k}-\sum_{k=1}^{n} c_{k}-n m e\right)} .
\end{aligned}
$$

We already established that the denominator is positive, so $d Q / d \bar{E}>0$ showing that the increase in the emission standard always has an increasing effect on the industry output as well as on the total emission level.

From (11), we have that

$$
\bar{E}=\frac{[2+(n-1) \gamma] Q^{2}-\left[\sum_{k=1}^{n}\left(a_{k}-c_{k}\right)-n m e\right] Q}{(n-1) m}
$$

which is a convex parabola in $Q$ with all other parameters assumed to be fixed. Its roots are zero and

$$
Q^{*}=\frac{\sum_{k=1}^{n}\left(a_{k}-c_{k}\right)-n m e}{2+(n-1) \gamma} .
$$

The cases of $Q^{*}>0$ and $Q^{*}<0$ are illustrated in Figures $1(\mathrm{a})$ and $1(\mathrm{~b})$.

With positive values of $\bar{E}>0, Q$ increases in $\bar{E}$ in both cases illustrating the conclusion based on relation (19).

\section{Dynamic Extensions and Stability Analysis}

Assume the government has a time delay $\tau>0$ in posing penalty or giving reward to the firms. If the firms use gradient adjustment, the adjustment process in discrete time scales is

$$
\begin{aligned}
& q_{k}(t+1)=q_{k}(t) \\
& \quad+S_{k}\left[\left(a_{k}-2 q_{k}(t)-\gamma Q(t)+\gamma q_{k}(t)-c_{k}\right)\right. \\
& \left.\quad-g_{k}(t-\tau)\right]
\end{aligned}
$$

that turns to be the following form in continuous time scales

$$
\begin{aligned}
& \dot{q}_{k}(t)=S_{k}\left[\left(a_{k}-2 q_{k}(t)-\gamma Q(t)+\gamma q_{k}(t)-c_{k}\right)\right. \\
& \left.-g_{k}(t-\tau)\right]
\end{aligned}
$$

where

$$
\begin{aligned}
& g_{k}(t-\tau)=m\left(e-\frac{Q(t-\tau)-q_{k}(t-\tau)}{E}\right) \\
& Q(t-\tau)^{2} \\
& \text { for } k=1,2, \ldots, n .
\end{aligned}
$$

In the literature best response dynamics are also used frequently; however, for linear systems, they are equivalent with gradient adjustment processes [10]. We will first examine discrete time dynamics and then continuous time dynamics to detect the stability conditions. 


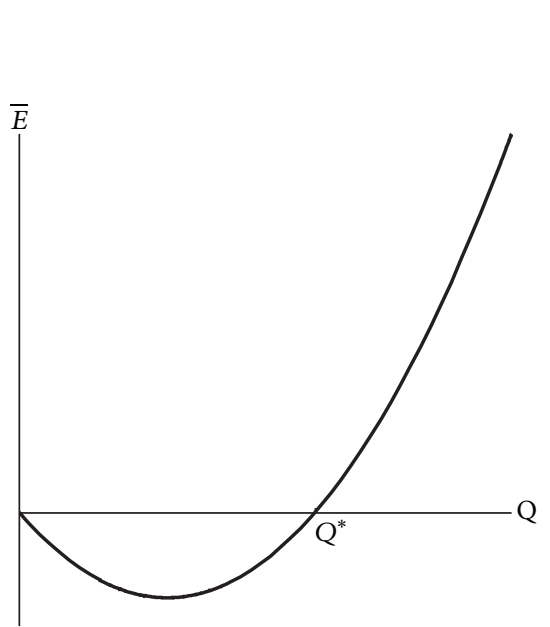

(a) $Q^{*}>0$

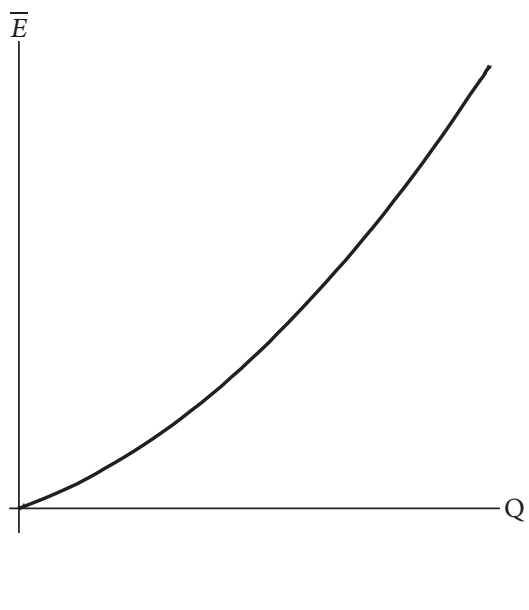

(b) $Q^{*}<0$

FIgURE 1: Relation of $Q$ and $\bar{E}$.

4.1. Discrete Time Model. When discrete time scales are adopted, the government delay is a positive integer $\tau$. Let $f_{k}^{D}$ denote the right-hand side of (22); then

$$
\begin{aligned}
\frac{\partial f_{k}^{D}}{\partial q_{k}(t)} & =1+\left(-S_{k}(2-\gamma)\right), \\
\frac{\partial f_{k}^{D}}{\partial q_{k}(t-\tau)} & =-\frac{S_{k} m \bar{E}}{Q(t-\tau)^{2}}<0, \\
\frac{\partial f_{k}^{D}}{\partial Q(t)} & =-S_{k} \gamma<0, \\
\frac{\partial f_{k}^{D}}{\partial Q(t-\tau)} & =\frac{S_{k} m \bar{E}\left[2 q_{k}(t-\tau)-Q(t-\tau)\right]}{Q(t-\tau)^{3}}<0,
\end{aligned}
$$

if there is no dominant firm. Let these derivatives be denoted by $1+A_{k}, B_{k}, C_{k}$ and $D_{k}$, respectively; then the linearized equation has the following form, where $q_{k}$ and $Q$ are now their distances from equilibrium levels:

$$
\begin{aligned}
q_{k}(t+1)= & \left(1+A_{k}\right) q_{k}(t)+C_{k} Q(t)+B_{k} q_{k}(t-\tau) \\
& +D_{k} Q(t-\tau) \quad \text { for } k=1,2, \ldots, n .
\end{aligned}
$$

It is challenging to consider this $n$-dimensional system of delay difference equations. For the sake of simplicity, we make the following two assumptions.

Assumption 2. The firms are identical in sense that they have the same adjustment coefficients, the same reservation prices, and the same marginal costs:

$$
\begin{aligned}
& S_{1}=S_{2}=\cdots=S_{n}=S, \\
& a_{1}=a_{2}=\cdots=a_{n}=a, \\
& c_{1}=c_{2}=\cdots=c_{n}=c .
\end{aligned}
$$

Assumption 3. The firms have the same initial level of output:

$$
q_{1}(0)=q_{2}(0)=\cdots=q_{n}(0)=q(0) .
$$

Under these assumptions, the equilibrium levels of the firms are identical and dynamic equation (22) generates identical trajectories of $q_{k}(t)$ for $k=1,2, \ldots, n$. In addition, the coefficients $A_{k}, B_{k}, C_{k}$, and $D_{k}$ are also identical, $Q(t)=$ $n q(t)$ and $Q(t-\tau)=n q(t-\tau)$. The delay difference equation is now written as

$$
q(t)=\alpha q(t-1)+\beta q(t-\tau) \quad \text { for } \tau \geq 2
$$

where

$$
\begin{aligned}
& \alpha=1+A+n C, \\
& \beta=B+n D .
\end{aligned}
$$

It should be noticed that, at the equilibrium,

$$
\begin{aligned}
& A+n C=-S[(n-1) \gamma+2]<0, \\
& B+n D=-\frac{(n-1) m S \bar{E}}{Q^{2}}<0 .
\end{aligned}
$$

Equation (29) is a linear delay difference equation. A lot of effort has been devoted to detect the corresponding stability condition. According to Čermák [11], there are three versions of the stability condition so far. The following is Theorem 4 of Čermák [11] that presents a necessary and sufficient condition on $\alpha$ and $\beta$ for the asymptotic stability of (29). (Theorem 4 is a slightly modified version of the original theorem of Papanicolaou [12]. Variables $\alpha$ and $\beta$ are introduced to be consistent with the notation of this paper.)

Theorem 4. The zero solution of (29) is asymptotically stable if and only if $(\alpha, \beta)$ is an internal point of the finite area bounded by two lines

$$
\begin{array}{r}
\alpha+\beta=1, \\
-\alpha+(-1)^{\tau} \beta=1
\end{array}
$$




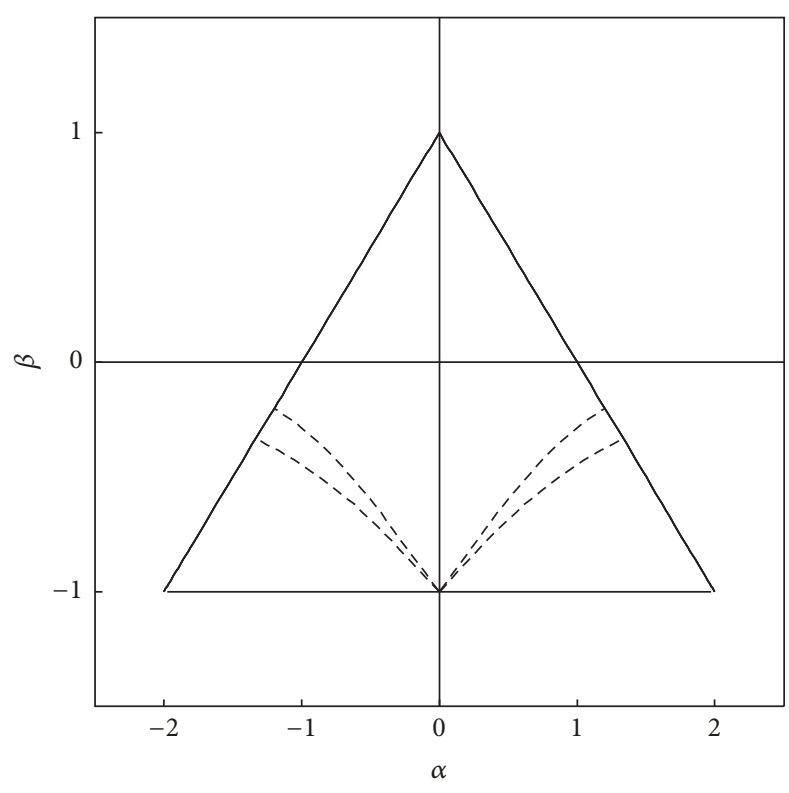

(a) $\tau=2,4,6$

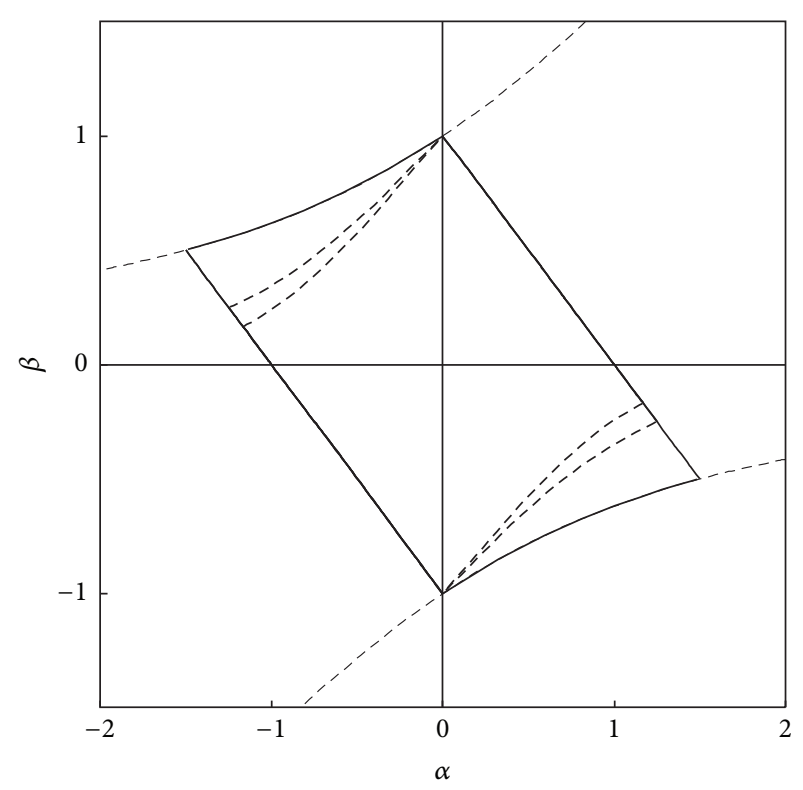

(b) $\tau=3,5,7$

Figure 2: Asymptotic stability regions.

and two transcendental curves

$$
\begin{aligned}
& \alpha=\frac{\sin (\tau \theta)}{\sin [(\tau-1) \theta]}, \\
& \beta=-\frac{\sin (\theta)}{\sin [(\tau-1) \theta]}, \\
& \alpha=-\frac{\sin (\tau \theta)}{\sin [(\tau-1) \theta]}, \\
& \beta=(-1)^{\tau-1} \frac{\sin (\theta)}{\sin [(\tau-1) \theta]}
\end{aligned}
$$

where $\theta \in(0, \pi / \tau)$.

In Figure 2(a), three stability regions corresponding to $\tau=2,4$ and 6 are illustrated. (We can construct the stability region for any number of $\tau$ by applying Theorem 4.) In particular for $\tau=2$, the stability region becomes the isosceles triangle, the right side is described by $\beta=1-\alpha$, the left side by $\beta=1+\alpha$, and the base by $\beta=-1$. For $\tau=4,6$, the horizontal base becomes a positive-sloping concave dashed curve for $\alpha>0$ and a negative-sloping concave dashed curve for $\alpha<0$, leading to a quadrilateral-wise stability region. As the value of $\tau$ increases with an increment of 2 , the dashed curves shift upward and thus the stability region shrinks. On the other hand, in Figure 2(b), three stability regions corresponding to $\tau=3,5$ and 7 are illustrated. The stability regions with odd $\tau$ have parallelogram-wise shape with nonlinear upper and lower sides which are the concave and convex dotted curves. As $\tau$ increases, the upper side rotates downward-around the vertical axis and the lower side rotates upward-around the vertical axis. As a result increasing $\tau$ has a decreasing effect on the stability region. The right and left sides described by $\beta=1-\alpha$ and $\beta=-1-\alpha$ are not affected by a change in the value of $\tau$. In short, we summarize the properties of the stability region as follows:

(1) The stability regions with even $\tau$ are slightly different than those with odd $\tau$.

(2) Increasing $\tau$ decreases the stability region.

(3) The stability region is the intersection of two subregions: one is delay-independent and the other is delay-dependent.

We can derive the characteristic polynomial by looking for the solution in a special form, which is the power function:

$$
q(t)=\lambda^{t} u
$$

Substituting this solution into (29), we have, after simplification,

$$
\lambda^{\tau}-\alpha \lambda^{\tau-1}-\beta=0
$$

In principle, finding the locations of $\lambda$ yields the stability conditions for the zero solution of the delay difference equation. In particular, if $\tau=2$, then the characteristic equation (36) is quadratic:

$$
\lambda^{2}-\alpha \lambda-\beta=0
$$

Asymptotic stability is guaranteed by the following conditions [10]:

$$
\begin{aligned}
1+\alpha-\beta & >0 \\
1-\alpha-\beta & >0 \\
-\beta & <1 .
\end{aligned}
$$


These three conditions construct the isosceles triangle in Figure 2(a). Returning to the model parameters, (31) and (32) immediately imply that the second condition is clearly satisfied:

$$
1-\alpha-\beta=-[(A+n C)+(B+n D)]>0 .
$$

Since the first condition is

$$
1+\alpha-\beta=2+(A+n C)-(B+n D)>0,
$$

using (31) and (32), it reduces to

$$
(B+n D)-(A+n C)=\frac{n S}{Q}(\alpha-C-m e)<2
$$

and the third condition has the form

$$
-\beta=(n-1) \frac{m S \bar{E}}{Q^{2}}<1 .
$$

In the case with $\tau=2$, we notice the following:

(a) If $a-C-m e<0$, then (41) holds and (42) is satisfied if $Q$ is sufficiently large or the speed of adjustment $S$ is sufficiently small.

(b) If $a-C-m e \geq 0$, then the discrete system is stable if $Q$ is sufficiently large or the value of $S$ is sufficiently small.

An equivalent condition can be given by rewriting (41) and (42) as

$$
\begin{aligned}
& Q>\frac{n S}{2}(a-C-m e), \\
& Q>\sqrt{(n-1) m S \bar{E}} .
\end{aligned}
$$

Letting

$$
Q^{*}=\max \left[\frac{n S}{2}(a-C-m e), \sqrt{(n-1) m S \bar{E}}\right],
$$

the stability condition becomes

$$
P\left(Q^{*}\right)<0
$$

where $P(Q)$ denotes the left-hand side of (11).

If $\tau=3$, then characteristic equation (36) is cubic:

$$
\lambda^{3}-\alpha \lambda^{2}-\beta=0
$$

The stability conditions (except trivial one) are as follows [13]:

$$
\begin{gathered}
1-\alpha-\beta>0, \\
1+\alpha+\beta>0, \\
1+\alpha \beta-\beta^{2}>0 .
\end{gathered}
$$

These three inequalities construct the parallelogram-wise stability region. The parallel negative-sloping lines are described by the first two conditions of (47) with the equality. In the same way, replacing the inequality of the third condition of (47) with the equality and solving for $\beta$ yields two solutions:

$$
\begin{aligned}
& \beta=\frac{\alpha+\sqrt{\alpha^{2}+4}}{2}, \\
& \beta=\frac{\alpha-\sqrt{\alpha^{2}+4}}{2} .
\end{aligned}
$$

The first equation presents a positive-sloping convex dotted curve in Figure 2(b), some part of which overlaps the upper side of the stability region whereas the second equation gives a positive-sloping concave dotted curve, some part of which overlaps the lower side.

As is already seen, the first condition of (47) always holds. The second condition can be written as

$$
1+\alpha+\beta=2-S\left[(n-1)\left(\gamma+\frac{m \bar{E}}{Q^{2}}\right)+2\right]>0 .
$$

The inequality holds for sufficiently small $S, n, \bar{E}$ and sufficiently large $Q$. The third condition is equivalent to

$$
\alpha<\beta-\frac{1}{\beta} .
$$

since $\beta<0$ from (32). The right-hand side is positive for $\beta>-1$ with which this inequality holds as $\alpha<0$. The absolute value of $\beta$ can be small when $S, n$, and $\bar{E}$ are small and/or $Q$ is large. Therefore, the stability conditions for $\tau=3$ might be satisfied if $S, n$, and $\bar{E}$ are sufficiently small and/or $Q$ is sufficiently large. For larger $\tau$ values, the stability conditions in terms of the model parameters become even more complicated although the stability regions can be visualized due to Theorem 4 .

4.2. Continuous Time Model. We now draw attention to the dynamic equation (23) that is a nonlinear delay differential equation. If the right-hand side of (23) is denoted by $f_{k}^{C}$, the followings are easily verified:

$$
\begin{gathered}
\frac{\partial f_{k}^{C}}{\partial q_{k}(t)}=\frac{\partial f_{k}^{D}}{\partial q_{k}(t)}-1=A_{k}, \\
\frac{\partial f_{k}^{C}}{\partial q_{k}(t-\tau)}=\frac{\partial f_{k}^{D}}{\partial q_{k}(t-\tau)}=B_{k}, \\
\frac{\partial f_{k}^{C}}{\partial Q(t)}=\frac{\partial f_{k}^{D}}{\partial Q(t)}=C_{k}, \\
\frac{\partial f_{k}^{C}}{\partial Q(t-\tau)}=\frac{\partial f_{k}^{D}}{\partial Q(t-\tau)}=D_{k} .
\end{gathered}
$$

Under Assumptions 1 and 2, the firms become symmetric and linearizing (23) in the neighborhood of the equilibrium yields

$$
\dot{q}(t)=(\alpha-1) q(t)+\beta q(t-\tau) .
$$

This is a linear delay differential equation and its asymptotic behavior depends on the location of the eigenvalues. As usual 
in the theory of delay differential equations [14], we look for the solution in exponential form:

$$
q(t)=e^{\lambda t} u
$$

then we have the characteristic equation:

$$
\lambda-(\alpha-1)-\beta e^{-\lambda \tau}=0 .
$$

As $\tau=0$, when the equation is without delay, the characteristic root is negative:

$$
\lambda=-(1-\alpha-\beta)<0
$$

implying that the system is asymptotically stable. Stability switch occurs if $\lambda=i \omega$ with some $\omega>0$, when

$$
i \omega-(\alpha-1)-\beta(\cos \omega \tau-i \sin \omega \tau)=0 .
$$

Separating the real and imaginary parts,

$$
\begin{aligned}
-(\alpha-1)-\beta \cos \omega \tau & =0, \\
\omega+\beta \sin \omega \tau & =0 .
\end{aligned}
$$

So

$$
\omega^{2}=\beta^{2}-(\alpha-1)^{2}=(\beta+\alpha-1)(\beta-\alpha+1)
$$

where $\beta+\alpha-1<0$ is already confirmed. In our case

$$
\beta-\alpha+1=S\left\{(n-1)\left[\gamma-\frac{m \bar{E}}{Q^{2}}\right]+2\right\}
$$

The sign of the braced terms on the right-hand side of (60) is ambiguous. Since $\beta+\alpha-1<0$, there is no solution for $\omega$ if the right-hand side is nonnegative, and therefore there is no stability switch. If it is negative, then there is a unique value of $\omega$,

$$
\omega=\sqrt{\beta^{2}-(\alpha-1)^{2}} .
$$

Notice that this is the case if

$$
\gamma<\frac{m \bar{E}}{Q^{2}}-\frac{2}{n-1}
$$

which occurs if environmental standard and/or penalty/ reward factor is large or the industry output and/or substitutability factor is small.

Hopf bifurcation is used to find the direction of the stability switch. Let $\tau$ be the bifurcation parameter and consider $\lambda$ as function of $\tau$ : $\lambda=\lambda(\tau)$. By implicitly differentiating (54) with respect to $\tau$, we have

$$
\frac{d \lambda}{d \tau}-\beta e^{-\lambda \tau}\left(-\lambda-\frac{d \lambda}{d \tau} \tau\right)=0
$$

Solving this for the derivative presents

$$
\frac{d \lambda}{d \tau}=\frac{-\beta \lambda e^{-\lambda \tau}}{1+\beta \tau e^{-\lambda \tau}}=\frac{-\lambda[\lambda-(\alpha-1)]}{1+\tau[\lambda-(\alpha-1)]},
$$

where (54) is used from the first line to the second line. When $\lambda=i \omega$,

$$
\begin{aligned}
\left.\frac{d \lambda}{d \tau}\right|_{\lambda=i \omega} & =\frac{-i \omega[i \omega-(\alpha-1)]}{1+\tau[i \omega-(\alpha-1)]} \\
& =\frac{\omega^{2}+i \omega(\alpha-1)}{[1-\tau(\alpha-1)+i \tau \omega]} \frac{[1-\tau(\alpha-1)-i \tau \omega]}{[1-\tau(\alpha-1)-i \tau \omega]} \\
& =\frac{\omega^{2}+i\left[\omega(\alpha-1)(1-\tau(\alpha-1))-\tau \omega^{3}\right]}{[1-\tau(\alpha-1)]^{2}+(\tau \omega)^{2}} .
\end{aligned}
$$

Hence the real part is

$$
\operatorname{Re}\left[\left.\frac{d \lambda}{d \tau}\right|_{\lambda=i \omega}\right]=\frac{\omega^{2}}{[1-\tau(\alpha-1)]^{2}+(\tau \omega)^{2}}>0,
$$

showing that the sign of the real part changes from negative to positive as the value of $\tau$ increases from zero, so stability is lost. That is, at the smallest stability switching point, stability is lost and it cannot be regained later. If there is positive solution for $\omega$, then $|\beta|>|\alpha-1|$, and since from (57) and (58) we know that $\sin \omega \tau>0$ and $\cos \omega \tau<0$, the smallest (critical) value of $\tau$ is the following:

$$
\tau^{*}=\frac{1}{\sqrt{\beta^{2}-(\alpha-1)^{2}}} \cos ^{-1}\left(-\frac{\alpha-1}{\beta}\right) .
$$

At the critical value of $\tau$ there is the possibility of the birth of limit cycles. From (60), we know that the system is always asymptotically stable if

$$
m \bar{E}(1-n)+[(n-1) \gamma+2] Q^{2} \geq 0 .
$$

From (11), the left-hand side equals

$$
\left(\sum_{k=1}^{n} a_{k}-\sum_{k=1}^{n} c_{k}-n m e\right) Q=n(a-c-m e) Q
$$

so the system is stable if multiplier of $Q$ is nonnegative. (The inequality $a_{k}>c_{k}$ is usually assumed to have a positive level of output of firm $k$ in a standard Cournot oligopoly model. So if $m e$ is small, then $a_{k} \geq c_{k}+m e$. However, $m e$ can be large enough to have $a_{k}<c_{k}+m e$.) Notice that the multiplier of $Q$ is the total marginal profit of the firms at zero environmental standard when all firms have zero production levels.

\section{Concluding Remarks}

This paper examines $n$-firm oligopolies with product differentiation when the firms face penalties or rewards depending on the amounts of their pollution levels. The government selects an emission standard for the entire industry, and based on the specific technology and output of each firm, the government determines its maximum allowed emission level. The amount of penalty or that of the reward is determined by the difference of the actual emission level and the maximum allowed amount. This simple mathematical model can be considered as the counterpart of models with ambient 
pollution charges discussed earlier in the literature. The existence of the unique interior equilibrium is first proved, and then the effects caused by changes in the strategic or policy variables, $m$ and $\bar{E}$, of the government are examined. We establish that an increase in the value of $m$ has an increasing effect on the total pollution level if the emission standard $\bar{E}$ is sufficiently large; otherwise the opposite effect occurs. An increase in the value of $\bar{E}$ always has an increasing effect on the total pollution level of the industry. Dynamic extensions are introduced and the stability of the equilibrium is examined with both discrete and continuous time scales. In the case of discrete time scales, the different values of the delay length are assumed in the penalty and reward terms and in the continuous time scales, a positive delay is introduced. In the case of discrete time scales the equilibrium is stable if either the industry output is sufficiently large or the common speed of adjustment of the firms is sufficiently small. In the case of continuous time scales the equilibrium is always stable if the total marginal profit of the firms at zero output levels is nonnegative; otherwise it is stable if the length of the delay is sufficiently small. At the critical value of the delay Hopf bifurcation occurs.

Symmetric firms are assumed for mathematical simplicity, in which case the equilibrium could be given analytically; however, in the nonsymmetric case, it is not possible making the further analysis much more complicated. This issue will be the subject of our next project. We will also examine the possibility of technology changes. In this case the additional costs and reduced penalties have to be compared.

\section{Conflicts of Interest}

The authors declare that there are no conflicts of interest regarding the publication of this paper.

\section{Acknowledgments}

The authors highly appreciate the financial supports from the MEXT-Supported Program for the Strategic Research Foundation at Private Universities 2013-2017, the Japan Society for the Promotion of Science (Grant-in-Aid for Scientific Research (C), 25380238, 26380316, and 16K03556), and Chuo University (Joint Research Grant). The usual disclaimer applies.

\section{References}

[1] P. B. Downing and L. J. White, "Innovation in pollution control," Journal of Environmental Economics and Management, vol. 13, no. 1, pp. 18-29, 1986.

[2] C. Jung, K. Krutilla, and R. Boyd, "Incentives for advanced pollution abatement technology at the industry level: an evaluation of policy alternatives," Journal of Environmental Economics and Management, vol. 30, no. 1, pp. 95-111, 1996.

[3] J.-P. Montero, "Permits, standards, and technology innovation," Journal of Environmental Economics and Management, vol. 44, no. 1, pp. 23-44, 2002.
[4] K. Okuguchi and F. Szidarovszky, "Existence of Nash-Cournot equilibrium in oligopoly games with pollution treatment costsharing," Pure Mathematics and Applications, vol. 13, pp. 455$462,2002$.

[5] K. Okuguchi and F. Szidarovszky, "Environmental R\&D in Cournot oligopoly with emission or performance standards," Pure Mathematics and Applications, vol. 18, pp. 111-118, 2007.

[6] K. Segerson, "Uncertainty and incentives for nonpoint pollution control," Journal of Environmental Economics and Management, vol. 15, no. 1, pp. 87-98, 1988.

[7] S. Ganguli and S. Raju, "Perverse environmental effects of ambient charges in a Bertrand duopoly," Journal of Environmental Economics and Policy, vol. 1, no. 3, pp. 289-296, 2012.

[8] S. Raju and S. Ganguli, "Strategic firm interaction, returns to scale, environmental regulation and ambient charges in a Cournot duopoly," Technology and Investment, vol. 4, no. 2, pp. 113-122, 2013.

[9] A. Matsumoto, F. Szidarovszky, and M. Yabuta, "Environmental effects of ambient charge in Cournot oligopoly," Journal of Environmental Economics and Policy, vol. 7, no. 1, pp. 41-56, 2018.

[10] G. I. Bischi, C. Chiarella, M. Kopel, and F. Szidarovszky, Nonlinear Oligopolies: Stability and Bifurcations, Springer, Berlin, Germany, 2010.

[11] J. Čermák, "Stability conditions for linear delay difference equations: a survey and perspective," Tatra Mountains Mathematical Publications, vol. 63, pp. 1-29, 2015.

[12] V. G. Papanicolaou, "On the asymptotic stability of a class of linear difference equations," Mathematics Magazine, vol. 69, no. 1, pp. 34-43, 1996.

[13] R. W. Farebrother, "Simplified Samuelson conditions for cubic and quartic equations," The Manchester School, vol. 41, no. 4, pp. 396-400, 1973.

[14] R. Bellman and K. Cook, Differential-difference Equations, Academic Press, New York, NY, USA, 1956. 


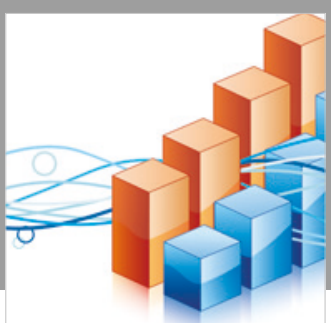

Advances in

Operations Research

\section{-n-m}
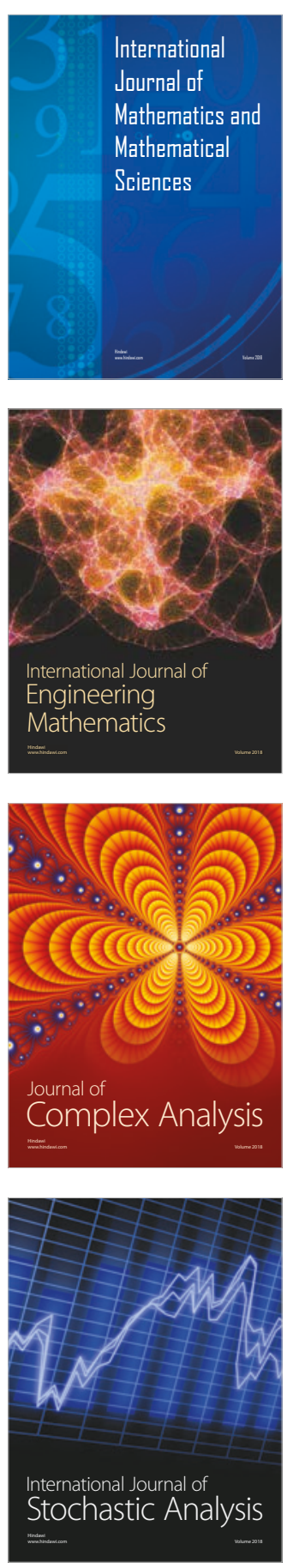
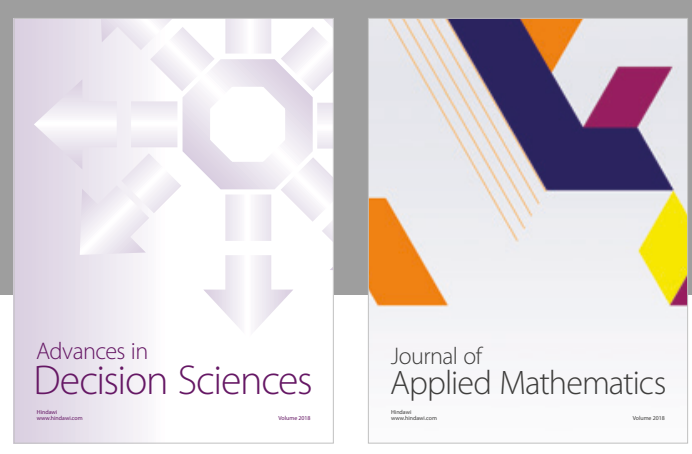

Journal of

Applied Mathematics
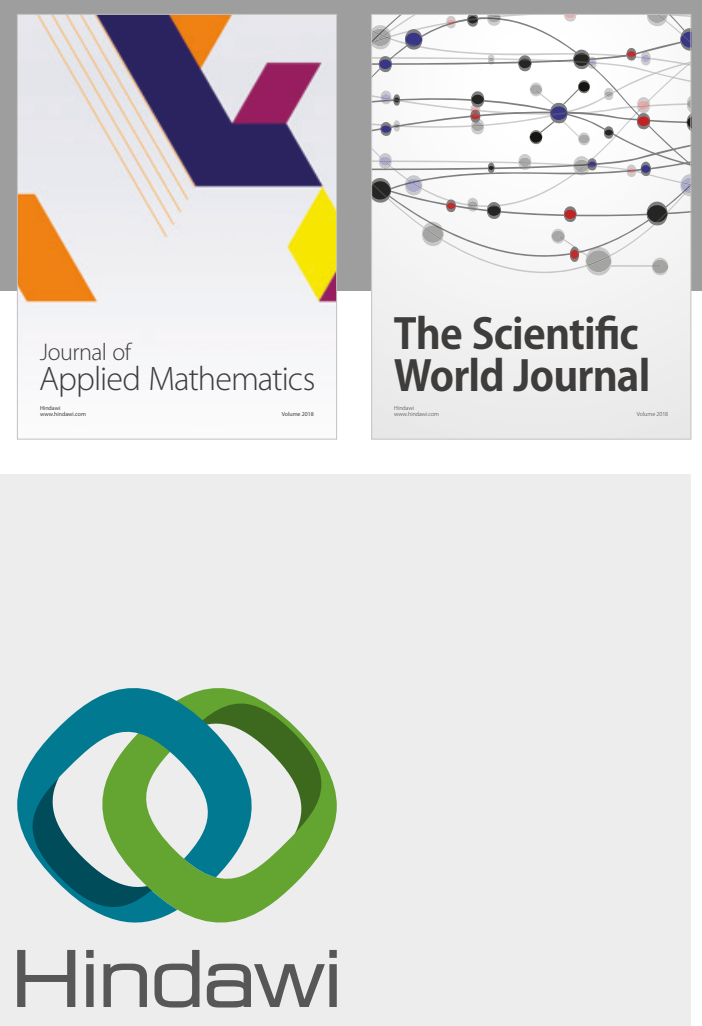

Submit your manuscripts at

www.hindawi.com

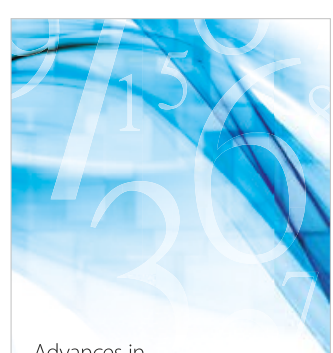

Advances in
Numerical Analysis
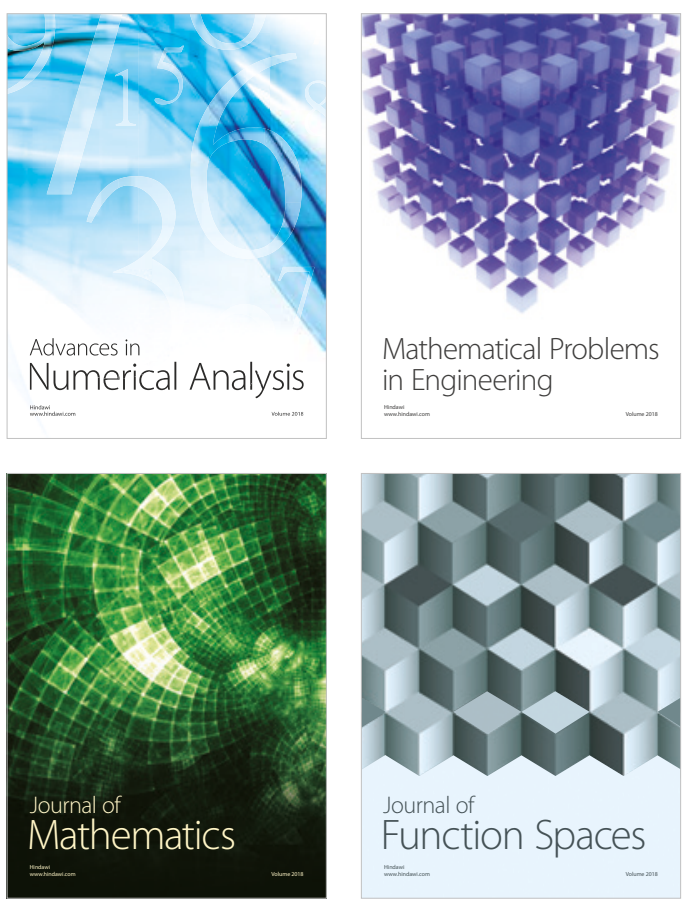

Mathematical Problems in Engineering

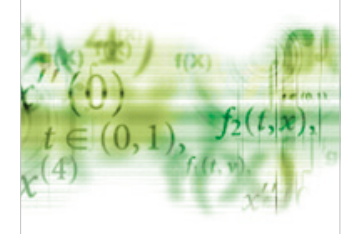

International Journal of

Differential Equations

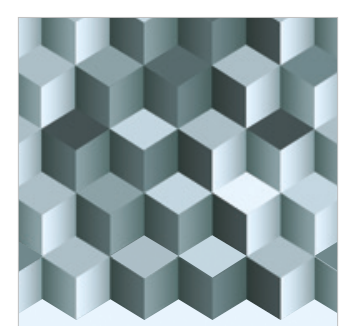

Journal of

Function Spaces

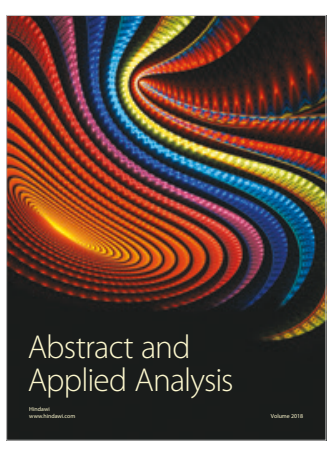

The Scientific

World Journal

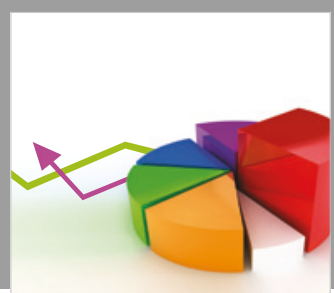

Journal of

Probability and Statistics
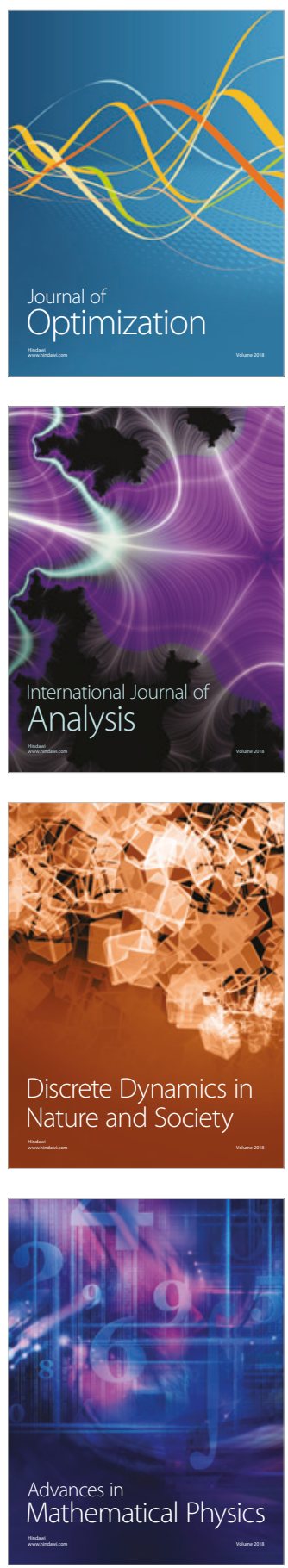